\title{
Covalent adaptable networks with tunable exchange rates based on reversible thiol-yne cross-linking
}

\author{
Niels Van Herck, ${ }^{[a]}$ Diederick Maes, ${ }^{[a]}$ Kamil Unal, ${ }^{[a, b]}$ Marc Guerre, ${ }^{[a, c]}$ Johan M. Winne, ${ }^{*[b]}$ and Filip E. \\ Du Prez ${ }^{*[a]}$
}

\begin{abstract}
The design of covalent adaptable networks (CANs) relies on the ability to trigger the rearrangement of bonds within a polymer network. Here, we introduce simple activated alkynes as versatile reversible cross-linkers for thiol functionalities. The click-like thiol-yne cross-linking reaction readily enables network synthesis from polythiols, through a double Michael addition with a reversible and tunable second addition step. The resulting thioacetal cross-linking moieties serve as robust but dynamic linkages, offering a versatile platform for the development of CANs. A series of different activated alkynes have been synthesized and systematically probed for their ability to produce dynamic thioacetal linkages, both in kinetic studies of small molecule models, as well as in stress relaxation and creep measurements on thiol-yne-based CANs. The results are further rationalized by DFT calculations and show that the bond exchange rates can be significantly influenced by the choice of the activated alkyne cross-linker.
\end{abstract}

\section{Introduction}

Covalent adaptable networks (CANs) are polymer materials that combine the strength of covalently crosslinked materials, such as high chemical resistance and dimensional stability, with the reprocessability of thermoplastic polymers. ${ }^{[1-4]}$ These responsive polymer networks have the ability to reversibly rearrange their network topology through the action of dynamic exchanges. Upon application of an external stimulus (e.g., heat, light, etc.), the dynamic chemistry is activated to allow bond exchanges on a molecular level, which enables network plasticity and allows reshaping, reprocessing, stress relaxation, self-healing and imprinting.

An important characteristic of CANs is the underlying bond exchange mechanism as it ultimately determines the mechanical properties of a CAN during the applied stimulus. ${ }^{[3]}$ Reversible polymer networks or so-called dissociative CANs imply that a

[a] N. Van Herck, D. Maes, K. Unal, Dr. M. Guerre, Prof. Dr. F. E. Du Prez

Polymer Chemistry Research Group, Center of Macromolecular Chemistry $(\mathrm{CMaC})$, Departement of Organic and Macromolecular Chemistry, Faculty of Sciences, Ghent University

Krijgslaan 281 (S4-bis), 9000 Ghent, Belgium

E-mail: Filip.DuPrez@UGent.be

[b] K. Unal, Prof. Dr. J. M. Winne

Laboratory for Organic Synthesis, Department of Organic and Macromolecular Chemistry, Faculty of Sciences, Ghent University Krijgslaan 281 (S4-bis), 9000 Ghent, Belgium

E-mail: Johan.Winne@UGent.be

[c] Dr. M. Guerre

Laboratoire des IMRCP, Université de Toulouse, CNRS UMR 5623, Université Paul Sabatier, 118 route de Narbonne, 31062 Toulouse Cedex 9, France

Supporting information for this article is given via a link at the end of the document. bond-breaking event happens prior to the formation of a new bond (i.e., an elimination-addition mechanism), whereas bond exchange in permanent dynamic networks, also called vitrimers or associative CANs, is achieved through a bond-forming/bondbreaking sequence (i.e., an addition-elimination reaction). ${ }^{[5,6]}$ As a result, dissociative CANs can show a temporary decrease in network connectivity during the stimulus application, which may result in a sudden decrease of viscosity, while associative CANs or vitrimers, preserve their network integrity in all conditions and cannot undergo a gel-to-sol transition. ${ }^{[7]}$ In this regard, many different chemistries have now been applied or purposely developed for CANs. For example, typical dissociative reversible systems are based on furan-maleimide chemistry, ${ }^{[8]}$ alkoxyamine chemistry, ${ }^{[9]}$ triazolinedione-indole chemistry, ${ }^{[10-12]}$ phthalate monoester transesterification, ${ }^{[13]}$ etc. while typical associative reversible systems are based on transesterification, ${ }^{[14]}$ vinylogous urethane transamidation, ${ }^{[15-18]}$ transalkylation, ${ }^{[19,20]}$ allyl sulfide exchange, ${ }^{[21]}$ boroxine exchange, ${ }^{[22]}$ etc.

As part of our group's continuing interest towards CANs and the exploration and development of new reversible chemistry platforms for their design, we were inspired by the base-catalyzed dynamic equilibria of thiols and activated alkenes. Although thiolene reactions are long-known in polymer chemistry as click-like bond forming reactions, their reversible nature has only recently been explored in polymer chemistry by Konkolewicz and coworkers (Scheme 1a). ${ }^{[23]}$ Further inspired by recent work by Kalow and coworkers using thioacetals as versatile dynamic cross-links (Scheme 1b), ${ }^{[24]}$ we became intrigued by the possibility of using simple activated alkynes, such as alkynones, directly as cross-linkers for thiol-based monomers and polymers, in a straightforward way resulting in possibly highly dynamic thioacetal linkages, starting from very simple precursors (Scheme 1c).

$$
\text { a) Konkolewicz, thiol-ene cross-linkers (2016): }
$$

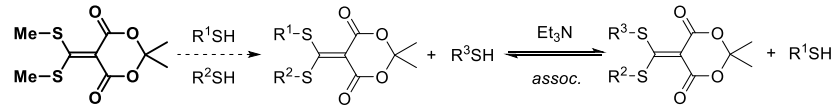

Scheme 1. Overview of dynamic thiol exchange chemistries for polymer networks: (a) Reversible thiol-ene Michael reaction of thiols and activated alkenes, (b) Ketene thioacetal exchanges through reversible Michael additions to a Meldrum's acid derivative, (c) Investigated reversible thiol-yne Michael reactions of thiols and activated alkynes. 
In fact, the base-promoted dynamics of alkynone-derived thioacetals (cf. Scheme 1c) was investigated on small molecule level by Anslyn and coworkers in 2012. ${ }^{[25]}$ In this encouraging prior study, it was shown that the dynamic thiol exchange of thioacetals via retro-Michael reactions (vinylogous thioesters) was possible, and can thus likely be tuned for interesting applications in the context of dynamic combinatorial libraries. ${ }^{[26]}$ Recently, there has been a surge in reports on CANs that rely on dynamic thiol exchange via reversible thiol-Michael chemistry, ${ }^{[23,27-29]}$ transthioesterification ${ }^{[30]}$ and reversible exchange at a Meldrum's acid alkylidene. ${ }^{[24]}$ As the currently available thiol-based dynamic chemistries either require exotic reagents, free thiols that are prone to side reactions (e.g., oxidation), long relaxation times or aqueous basic conditions to allow exchange, we envisaged that the thiol-yne-derived dynamic thioacetal links could thus provide a quite valuable and complementary addition to the growing toolbox of dynamic 'thiolclick' chemistries. ${ }^{[31-33]}$ The very specific two-step mechanism of the 'double' Michael addition on an ynone moiety studied in this work offers two important advantages over existing thiol-based dynamic cross-linking chemistries. From a fundamental or pure polymer chemistry point of view, it can be expected that both the kinetics and the thermodynamics of bonding and debonding can be controlled (i.e., tunability) through introduction of substituents on key position of the activated yne, offering options to control the rate of exchange and the degree of connectivity, thereby bridging the standard situation of permanent versus non-permanent dynamic networks. This chemistry thus presents a good testing ground for new ideas, with many handles to control reactivity. Furthermore, from a very practical or applied polymer chemistry point of view, monofunctional ynone- or ynoate-type compounds can be directly used as bivalent cross-linkers for thiol moieties, making their practical application and synthesis cost less challenging.

In this work, we thus aimed for the exploration of dynamic thiolyne cross-linking as a new platform for the dynamic exchange of thioacetal linkages. The most desirable reactivity profiles in terms of CANs were first explored using kinetic studies on model compounds, followed by stress relaxation measurements on material level. It was found that the exchange rate could be controlled through the judicious choice of the alkynone (or activated alkyne) cross-linker. The observed influence of the alkynone structure on the exchange rate was further evaluated by density functional theory (DFT) calculations, which confirmed and could rationalize the experimental observations. The obtained polymer networks were subjected to extensive mechanical, thermal, stress relaxation and reprocessing tests to demonstrate the desired dynamic behavior.

\section{Results and Discussion}

The key functional monomers and cross-linkers used in this work are activated alkyne moieties ( $\mathbf{X}$, Scheme 2) like conjugated alkynones, that can serve as double Michael acceptors. Such moieties are readily available or can be easily prepared from simple precursors. A base-catalyzed click-reaction with two thiols can then result in a thioacetal derivative of $\mathbf{X}$ (TA-X, Scheme 2). The basic catalyst promotes deprotonation of the thiol to its more nucleophilic thiolate form, which performs the first Michael addition ((1), Scheme 2) on the electron-deficient triple bond of the alkynone. This can yield both isomers $(E / Z)$ of the $\beta$-sulfido$\alpha, \beta$-unsaturated carbonyl or vinylogous thioester derivative of the alkynone $\mathbf{X}$ (VTE-X). ${ }^{[25,34]}$ Subsequently, this initial Michael adduct can act again as a Michael acceptor to allow a second thiol addition ((2), Scheme 2). In previous studies, this second addition has been shown to be up to 1000 times slower than the first addition. ${ }^{[35]}$ The resulting final product, the thioacetal derivative TA-X, can nevertheless be obtained in very high overall yields (Table 1), depending on the used catalyst. ${ }^{[32,35-37]}$ This doubleaddition reaction pathway was first verified and monitored by ${ }^{1} \mathrm{H}$ NMR spectra for the reaction between a simple alkynone, but3-yn-2-one ( $\mathbf{X}=\mathbf{1}$, Table 1), and 2 equivalents of butane-1-thiol (Figure S1). Using only 1 mol\% of 1,5,7-triazabicyclo[4,4,0]dec-5ene (TBD) as a catalyst, the desired thioacetal adduct (TA-1) could be synthesized within 10 minutes at room temperature in chloroform with $95 \%$ yield. In these studies, TBD was chosen as a non-nucleophilic, strong, non-volatile and lipophilic base that is compatible with polymer formulations and their envisaged thermal reprocessing, and also immediately gave very good results on small molecule level. ${ }^{[36]}$

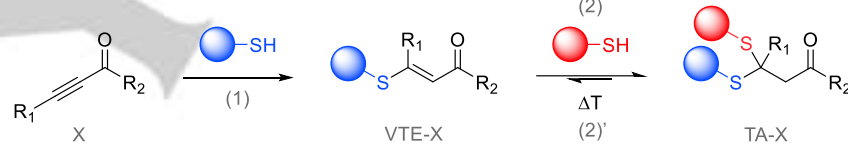

Scheme 2. Synthesis of a thioacetal adduct (TA-X) via the base-catalyzed double thiol-Michael addition on alkynone precursors (X). (VTE-X) represents the $\beta$-sulfido- $\alpha, \beta$-unsaturated carbonyl intermediate. Reaction (2)' corresponds to the dynamic thiol exchange occurring at high temperature.

Even though the forward reactions to yield the thioacetal adduct are spontaneous and thermodynamically favored, Anslyn reported that dynamic thiol exchange can happen upon the addition of alternative thiols and triethylamine in solution via the suspected retro-Michael reaction ((2)', Scheme 2). ${ }^{[25]}$ As our objective was to explore the utility of this reversible reaction in the context of CANs with dynamic thiol-yne derived thioacetal linkages, we first synthesized a series of small molecule TA-X compounds following the synthetic procedure depicted above ( $1 \mathrm{~mol} \%$ TBD in chloroform). With these thioacetals in hand, the dynamic thiol exchange was evaluated at elevated temperature by means of kinetic studies through the monitoring of reaction progress via ${ }^{1} \mathrm{H}$ NMR spectroscopy.

\section{Synthesis of model compounds}

To explore the scope and possible limitations of the thiol-yne dynamic chemistry platform for CANs, a wide series of alkynonetype double Michael acceptors $(\mathbf{X})$ was selected or prepared (Table 1) and then evaluated for their click-like ability to reliably form thioacetal cross-linkages (TA-X) (cf. Scheme 2). Only limited examples of double Michael additions to alkynones can be found in the literature, and the success of these reactions can be 
expected to be dependent on steric and electronic factors, where the second Michael reaction could theoretically favor either the VTE-X or the TA-X side. A high yield for this step is certainly possible, but is thus not guaranteed (e.g., see entry where $X=12$ ) The substituents directly bound to the alkyne function (Scheme 2, $R_{1}$ ), as well as the substituents directly bound to the carbonyl function $\left(R_{2}\right)$ were varied with the intent to probe both steric and electronic effects on the dynamic exchange. In this series of alkynones, but-3-yn-2-one $(\mathbf{X}=\mathbf{1})$ was arbitrarily selected as the benchmark to which all other alkynones will be compared. Alkynones 2, 13-15 were chosen to evaluate the effect of replacing the ketone functionality with an ester, amide, sulfonamide or sulfone, respectively, as these are also common moieties in polymer chemistry. Alkynones 3-9 represent derivatives of 1-phenylprop-2-yn-1-one, which allowed the exploration of the hypothetical control on the exchange through introduction of electronically (de)activating para-substituents. Alkynones 10-12 were chosen to evaluate the effect of substituents directly bound to the alkyne function. All alkynones were synthesized according to known literature procedures ${ }^{[38-43]}$ (see SI-file), except for alkynones 1, 2 and 12, which are commercially available.

Table 1. Overview of investigated alkynones $(\mathbf{X})$ with their corresponding experimental data on model compound and material level. $E_{a}$ : activation energy.

\begin{tabular}{|c|c|c|c|c|c|}
\hline Entry & $\mathbf{x}$ & $\begin{array}{l}\text { VTE-X[a] } \\
\text { Yield (\%) }\end{array}$ & $\begin{array}{c}\text { TA-X }^{[\mathrm{b}]} \\
\text { Yield (\%) }\end{array}$ & $\begin{array}{l}\text { Model study } \\
E_{a}(\mathrm{~kJ} / \mathrm{mol})\end{array}$ & $\begin{array}{c}\text { Rheology } \\
E_{a}(\mathrm{~kJ} / \mathrm{mol})\end{array}$ \\
\hline & 1 & 99 & 95 & $76.8 \pm 4.4$ & $137.4 \pm 1.8$ \\
\hline & 2 & 99 & 94 & /[d] & $197.8 \pm 2.5$ \\
\hline & 3 & 99 & 81 & $71.0 \pm 4.9$ & $128.4 \pm 0.3$ \\
\hline & 4 & $63^{[c]}$ & 92 & $76.2 \pm 2.1$ & $134.1 \pm 0.6$ \\
\hline & 5 & 99 & 81 & $74.4 \pm 3.8$ & $178.6 \pm 1.1$ \\
\hline & 6 & 99 & 93 & $75.4 \pm 2.6$ & $186.5 \pm 6.8$ \\
\hline & 7 & 92 & 88 & $66.2 \pm 14.9$ & $133.5 \pm 1.0$ \\
\hline & 8 & $83^{[c]}$ & 95 & $66.2 \pm 1.3$ & $124.3 \pm 1.1$ \\
\hline & 9 & $35^{[c]}$ & 97 & $75.1^{[e]}$ & $128.0 \pm 1.1$ \\
\hline & 10 & 99 & 25 & - & - \\
\hline & 11 & 93 & 0 & - & - \\
\hline & 12 & 99 & 34 & - & - \\
\hline & 13 & 99 & 27 & - & - \\
\hline & 14 & 99 & 6 & - & - \\
\hline
\end{tabular}

is,
"sis
15
85
[d]

[a] Yield after addition of 1 eq thiol. [b] Yield after addition of 2 eq thiol. [c] Isolated yield of VTE-X was lower than expected as a result of overreaction, generating the double thiol-Michael product TA-X. [d] $E_{a}$ could not be determined since no exchange was observed in the investigated temperature range. [e] $E_{a}$ could not be determined in a reliable way since exchange was too fast to collect kinetic data above $40^{\circ} \mathrm{C}$.

In a next step, the dynamics of the most readily obtained model compounds (TA-X) were evaluated via kinetic exchange studies. Table 1 shows overall very good yields for the first thiol-Michael addition to yield VTE-X compounds. For VTE-4, VTE-8 and VTE-9, the isolated yield was lower than expected only because of the very swift second Michael addition reaction, directly generating the double thiol-Michael product TA-X. However, the activated alkyne substrates 10-14 showed an incomplete second addition and were not further considered during the kinetic model studies or network synthesis, as they cannot serve as a thiol-yne-type crosslinker. This lower efficiency of the second thiol Michael reaction can be related to electronic and steric considerations. Thus, only the alkynones that gave highly efficient double thiolMichael additions with butane-1-thiol were further considered for our current investigations (see SI-file).

\section{Kinetic Model Studies}

To evaluate the dynamic behavior of the synthesized thioacetal adducts (TA-X), stock solutions containing TA-X, 1 mol\% TBD and an internal standard in deuterated benzene were prepared, followed by the addition of a fivefold excess of benzyl mercaptan as a competitive thiol to approach pseudo first-order conditions for the initial exchange rates (see SI for experimental details). During the exchange reaction, the butanethiol groups of the respective model compound were found to be gradually replaced by benzyl mercaptan groups, leading to the formation of a mixture of three different thioacetals: TA- $\mathbf{X}_{\mathbf{A A}}, \mathbf{T A}-\mathbf{X}_{\mathbf{A B}}, \mathbf{T A}-\mathbf{X}_{\mathbf{B B}}$, where $A A$, $A B$ and $B B$ describe the alkyl groups present in the thioacetal adduct: butane/butane, butane/benzyl and benzyl/benzyl respectively (Figure 1a for TA-1). The exchange reaction was monitored by following the characteristic ${ }^{1} \mathrm{H}$ NMR signal of the original thioacetal motif (TA-X $\mathbf{X}_{\mathrm{AA}}$, triplet at $4.35-4.50 \mathrm{ppm}$ ), which decreased in intensity, while the new thioacetal signals of the exchanged products (TA-X $\mathbf{X}_{\mathbf{A B}}, \mathbf{T A}-\mathbf{X}_{\mathbf{B B}}$ ) emerged upfield (Figure $1 \mathrm{~b}$ ). The remaining molar fraction of TA- $\mathbf{X}_{\mathbf{A A}}$ was followed over time to determine the rate constant $k$. This was repeated at different temperatures comprised between 40 and $70{ }^{\circ} \mathrm{C}$, which allowed creating an Arrhenius plot from which the activation energy $E_{a}$ could be deduced as the slope of the linear fit (see SIfile). 

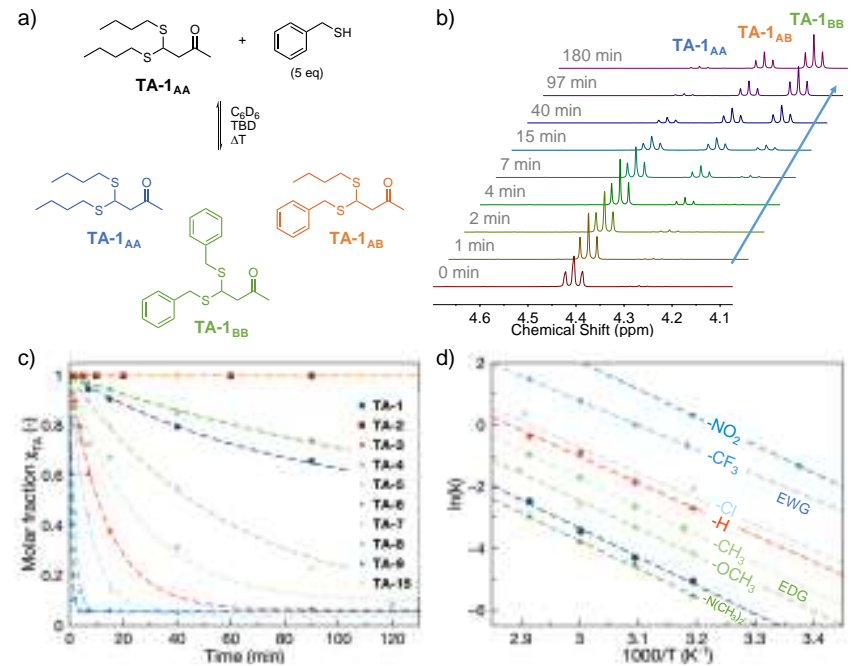

d)

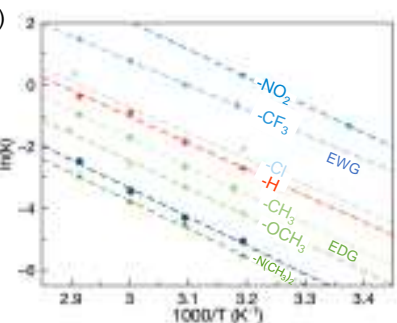

Figure 1. (a) Dynamic thiol exchange of TA-1 at elevated temperature with benzyl mercaptan, leading to 3 different exchange products: $\mathbf{T A}-\mathbf{1}_{\mathrm{AA}}, \mathbf{T A}-\mathbf{1}_{\mathbf{A B}}$ TA-1 $1_{\text {BB. }}$ (b) Evolution of characteristic ${ }^{1} \mathrm{H}$ NMR signals of the thioacetal motifs over time. (c) Overlay of remaining molar fraction of TA-X as a function of time during the exchange experiment at $40^{\circ} \mathrm{C}$. Dashed lines represent a fit with exponential decay. (d) Arrhenius plots of the reversible model compounds. Dashed lines represent a linear fit (the same color legend as in (c) applies). Chemical formulas represent the para-substituents of 1-phenylprop-2-yn-1-onederived compounds (TA-X, with $\mathbf{X}=\mathbf{3 - 9}$ )

Figure 1c shows an overlay of the remaining molar fractions of TA-X as a function of time during the exchange experiments at $40{ }^{\circ} \mathrm{C}$. Interestingly, the exchange kinetics were significantly influenced by the used model compound. Compared to the benchmark TA-1, some model compounds showed a remarkably faster dynamic thiol exchange, whereas others showed slower or even no exchange. From the Arrhenius plots in Figure 1d, similar activation energies $E_{a}$ within the range of $66-77 \mathrm{~kJ} / \mathrm{mol}$ were obtained for all reactions (Table 1). This could be expected since the exchange mechanism should be unchanged regardless of the used model compound. Nevertheless, a small trend could be observed in the data for the series of phenylpropynone-based model compounds (TA-X, with $\mathbf{X}=\mathbf{3 - 9}$ ). When the activation energy of TA-3 (no substituent on phenyl group) was taken as a reference $\left(E_{a}=71 \mathrm{~kJ} / \mathrm{mol}\right)$, the introduction of electron donating para-substituents $(X=4-6)$ added a penalty of $3-5 \mathrm{~kJ} / \mathrm{mol}$ to the energy barrier, whereas electron withdrawing para-substituents $(\mathbf{X}=7-9)$ reduced this barrier by $5 \mathrm{~kJ} / \mathrm{mol}$. As can be expected, the anionic transition state, i.e., when a thiolate anion is attacking the Michael acceptor, will be selectively stabilized over the neutral ground states, by the presence of electron withdrawing substituents on the aromatic ring conjugated to the receiving carbonyl function. However, considering that the small difference in $E_{a}$ is within experimental error for some model compounds, this interpretation of the temperature dependencies of the exchange rates remains speculative.

Nevertheless, the same trend in electronic activation is found to have a major effect on the actual exchange rates, spanning several orders of magnitude, as can be clearly seen in the
Arrhenius plot (Figure 1d). It is very clear from these data that electron withdrawing substituents accelerate the exchange significantly, whereas electron donating substituents showed the reverse effect (i.e., lower rate constants). The fastest exchange was observed for TA-9, where the introduction of a nitro group ($\mathrm{NO}_{2}$ ) allowed complete exchange within 2 minutes at $40^{\circ} \mathrm{C}$, even causing problematic sampling at higher temperatures. Conversely, the slowest exchange was observed for the analogous compound TA-6, where the only difference is the introduction of an electrondonating dimethylamino group $\left(-\mathrm{N}\left(\mathrm{CH}_{3}\right)_{2}\right)$, showing only $50 \%$ conversion after 4 hours at $40{ }^{\circ} \mathrm{C}$. The influence of parasubstituents on the exchange rate was also confirmed through Hammett analysis (Figure S2), which showed a strong positive linear correlation indicating an exchange mechanism via anionic intermediates. ${ }^{[4,45]}$

Another interesting observation was that model compounds TA-2 and TA-15 did not show exchange at the investigated temperature and time scale. Hence, they could be considered as practically irreversible, owing to the presence of the intrinsically less electron withdrawing ester or sulfone functional group. As intermediate conclusion, these results already indicated that the choice of the alkynone precursor is quite important with respect to the reversible nature of the thioacetal motif. Moreover, these experiments showed that the exchange rate could be significantly manipulated by means of the electronic substituents on the alkynone precursor.

For the design of CANs, the nature of the bond exchange mechanism is a key parameter since it dictates the viscoelastic profile of the resulting materials. The mechanism will also rely on the conditions and stoichiometry, and the reaction conditions for the model experiments described above may thus be a poor substitute for those found in thiol-yne-based polymer networks, where there may not be large amounts of free thiols present. Thus, to probe the thioacetal exchange in a more realistic setting, we also investigated whether the bond exchange can happen in the absence of free thiols. Therefore, two different thioacetal adducts were synthesized by reacting alkynone 1 with either butane-1thiol (TA-1 $\mathbf{A A}$ ) or benzyl mercaptan (TA-1 $\mathbf{B B}_{\mathbf{B B}}$. In a next step, these model compounds were mixed in a $1: 1$ stoichiometric ratio in deuterated benzene in the presence of TBD and heated to $70{ }^{\circ} \mathrm{C}$. ${ }^{1} \mathrm{H}$ NMR analysis revealed the gradual appearance of the mixed thioacetal moiety (i.e., TA-1 $\mathbf{A B}$ ) over time, which indicates that no free thiols are required to allow exchange (Figure S3). However, the exchange was found to proceed slower compared to the model experiments where an excess of free thiols was present. This result indicates that, while exchange rates are already fast at room temperature in our small molecule models, the situation inside a polymer network may be quite different as the exchange will also rely on statistics and diffusion limitations, therefore not directly reflecting the intrinsic chemical reaction rate.

\section{DFT Calculations}

Intrigued by the remarkable effect of the used alkynone precursor on the exchange kinetics, also density functional theory calculations were performed to confirm these observations. The Gaussian 16 package was used with the M06-2X/6-311+G(d,p) level of theory, which has been shown successful to describe the 
thermodynamics of the thiol-Michael addition (see SI for computational details). ${ }^{[46]}$ Using this theoretical method, the calculated Gibbs free energy profile of the double thiol-Michael addition on the alkynone compound $(\mathbf{X})$ could be modeled (see Figure S24-S37 and Table S2). The most crucial parameter coming out of this calculational approach is the relative Gibbs free energy of the transition state of the rate limiting step of the pathway $\left(\Delta G^{\circ}, \neq\right)$, referenced to the energetic ground state of the reactants and products.

In the graphical summary of these calculations (Figure 2), it can be seen that all thiol-Michael additions to the VTE-X intermediates are exergonic, with a thermodynamic driving force between only $15-20 \mathrm{~kJ} / \mathrm{mol}$. This readily indicates and confirms the reversible nature of this second bond formation step. The thermodynamic profiling of the individual reaction pathways is more challenging and subject to interpretation, but the relative Gibbs free energy difference for the modeled transition states, with reference to the ground states of the thioacetal adducts, indeed closely matches the trends observed in our kinetic experiments. Indeed, thiol exchange could only occur when the energetic barrier of $\Delta G^{\circ}$, is overcome to allow dissociation of the thioacetal (TA-X) into the vinylogous thioester (VTE-X) and thiolate anion. Therefore, this parameter is closely related to the activation energy $E_{a}$ as experimentally determined earlier during kinetic model studies.

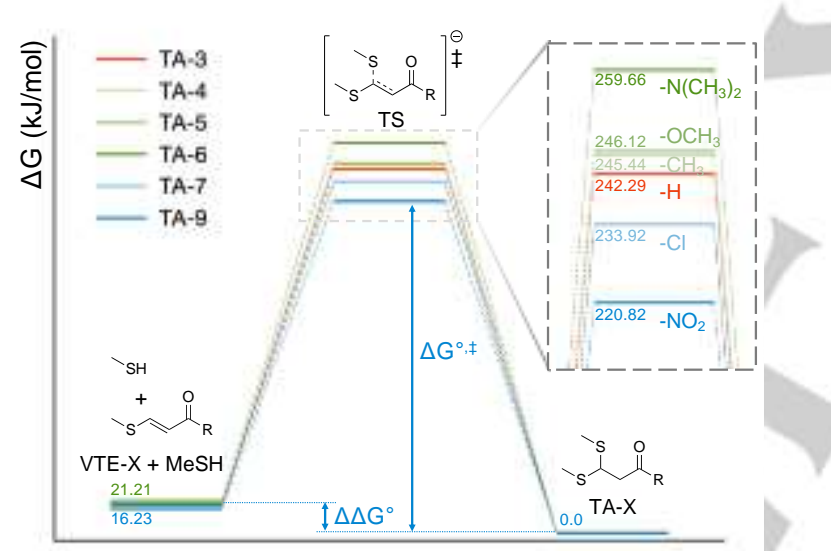

Figure 2. Calculated free energy diagram for the second thiol-Michael addition of methanethiol to VTE-X (with $\mathbf{X}=3-9$ ) with the formation of the TA-X product via the corresponding transition state (TS). The zoomed section shows the difference in $\Delta \mathrm{G}^{\circ}$, .

Interestingly, these calculations also provided a means to rationalize the incomplete formation of the desired thioacetal adducts from alkynones $\mathbf{1 0}$ and 13. In contrast to all other modeled VTE-X, the DFT-derived reaction Gibbs free energy for thiol addition to VTE-10 was found to favor the debonded state (see Table S2). Figure S33 shows that the reaction Gibbs free energy for 10 is calculated as slightly endergonic $\left(\Delta \Delta G_{10}^{\circ}=0.61\right.$ $\mathrm{kJ} / \mathrm{mol}$ ), indeed predicting an equilibrium mixture of VTE-10 and TA-10, as is experimentally observed. The same reasoning could not be applied to explain the incomplete formation of TA-13, as the reaction itself was found to be clearly exergonic by our calculation. However, the low yield for TA-13 could be attributed to the higher forward energetic barrier $\left(\Delta G^{\circ}, \neq\right)$, which may substantially slow down the second Michael reaction. The latter was also observed experimentally, as only $27 \%$ conversion of TA-13 was reached after 1 week at room temperature.

Our DFT model was unable to provide a rationalization for the distinct difference in reactivity observed for the seemingly nonreversible adducts TA-2 and TA-15, as comparable energy profiles were in fact obtained here (see SI). Thus, more advanced models may be required here.

\section{Synthesis of CANs}

Based on the outcome of the small molecule model study, the most interesting alkynones were selected for further investigation on a material's level. Alkynones $\mathbf{1}$ and 3-9 were expected to result in reversible cross-links in a dynamic polymer network, whereas alkynone $\mathbf{2}$ was assumed to give an irreversible material. Since alkynones have a functionality $(f)$ of 2 , the combination of an activated alkyne with any multifunctional thiol with $f \geq 3$ should directly result in a crosslinked polymer network. Originally, reaction of the alkynones with the commercially available pentaerythritol tetrakis(3-mercaptopropionate) was considered for the synthesis of polymer networks. However, this tetrafunctional thiol contains ester bonds, which are known to exchange via transesterification reactions in the presence of TBD. ${ }^{[47]}$ Therefore, this thiol was not suited for unambiguous design of dynamic materials that are solely relying on dynamic thiol exchange. Hence, an aliphatic trifunctional thiol (trithiol) ${ }^{[48]}$ was synthesized and used as a reference building block to produce dynamic polymer materials.

Thiol-yne polymer networks were prepared through reaction of the alkynone of interest with trithiol in the presence of TBD (see Scheme 3 and SI for experimental details). First, a vinylogous thioester prepolymer (VTEp-X) was prepared using 1 equivalent of trithiol with 3 equivalents of alkynone. In a second step, another equivalent of trithiol was added to the prepolymer to produce and fully cure the polymer network (CAN-X). As a result of this twophased protocol, also the relatively high exotherm associated with the double thiol-Michael addition could be better controlled, resulting in well-defined and reproducible network formation at completely stoichiometric conditions, theoretically containing no free thiols. The prepared networks were transparent and displayed a slight coloration depending on the used alkynone (Figure S4).

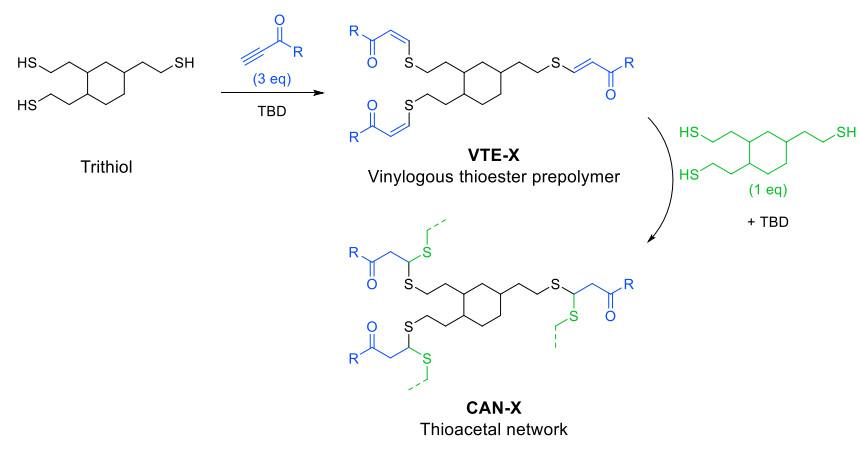


Scheme 3. Network synthesis via the reaction of trithiol (1 eq) with alkynone (3 eq) in the presence of TBD to yield the vinylogous thioester prepolymer (VTEp$\mathbf{X})$, followed by addition of trithiol (1 eq) to produce a thioacetal cross-linked network (CAN-X).

High-resolution magic angle spinning (HRMAS) ${ }^{1} \mathrm{H}$ NMR was used to confirm the presence of the thioacetal motif in the prepared polymer networks (Figure S5). Deuterated chloroform was selected as a non-reactive solvent providing very good swelling. Using this technique, a conversion of $85-90 \%$ could be estimated (residual VTE-signals integrating for $\sim 10 \%$ ), which is presumably caused by diffusion limitations in these highly crosslinked networks. Thus, the degree of polymerization should be well above the theoretical gel point (i.e., 71\%) according to the Flory-Stockmayer theory for a trifunctional $\left(A_{3}\right)$ and difunctional monomer $\left(B_{2}\right) \cdot{ }^{[49,50]}$ In order to further confirm network formation, swelling experiments were performed in tetrahydrofuran (THF) at room temperature to determine the soluble fraction for $\mathbf{C A N}-1$ and CAN-3 as representative networks (Table S1). Those networks showed a swelling ratio between $120-300 \%$ and a soluble fraction below $3 \%$.

\section{Thermal analysis of CANs}

Thermogravimetric analysis (TGA) of the prepared polymer networks demonstrated a single decomposition step with a maximum degradation rate at $\pm 330{ }^{\circ} \mathrm{C}$ for most of the networks (Figure 3a). CAN-4 and CAN-6 also showed an earlier decomposition step with an onset ( $5 \%$ weight loss) degradation temperature of $T_{d 5 \%}=176$ and $149^{\circ} \mathrm{C}$, respectively.

Differential scanning calorimetry (DSC) revealed the presence of a glass transition temperature $\left(T_{g}\right)$ that varied within the range of -16 to $52{ }^{\circ} \mathrm{C}$ depending on the used alkynone precursor (Figure 3b). Since all networks were prepared in the same way, this variable rigidity was attributed to the presence of freely rotating groups and conjugated $\pi$-systems in the alkynone compound. An increased $T_{g}$ was observed when a larger conjugated system was present in the matrix (cf. CAN-3 vs CAN-9). Interestingly, the simple presence of a (freely rotating) methoxy group in CAN-5 resulted in a very pronounced decrease of $T_{g}$. Besides the glass transition, an endotherm starting at $120^{\circ} \mathrm{C}$ was observed, which was attributed to the activation of the retro-Michael reaction at this temperature.
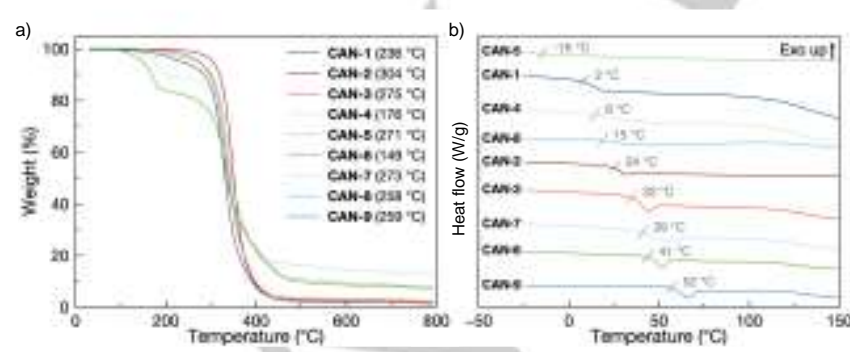

Figure 3. Thermal analysis of CANs. (a) Overlay of TGA thermograms. Values in brackets are the onset degradation temperatures at $5 \%$ weight loss $\left(T_{d 5}\right)$. (b) Overlay of DSC thermograms ( $2^{\text {nd }}$ heating). The onset $T_{g}$ is displayed above each trace.

\section{Rheological analysis of CANs}

To examine the viscoelastic properties of the dynamic networks, stress relaxation experiments were performed. During these measurements, a constant strain of $1 \%$ was applied and the relaxation modulus was followed as a function of time at different temperatures. Typical stress relaxation curves are presented in Figure $4 \mathrm{a}$ for CAN-1 and in Figures S6-S13 for all other CANs. As a result of dynamic bond rearrangement at high temperatures, full stress relaxation of the networks could be obtained. Next, relaxation times $(\tau)$ were determined at $37 \%(1 / \mathrm{e})$ of the normalized relaxation modulus $\left(G / G_{0}\right)$ according to the Maxwell model for viscoelastic fluids and plotted in an Arrhenius plot to obtain the corresponding viscous flow activation energy $E_{a}$ of 137 $\mathrm{kJ} / \mathrm{mol}$ for CAN-1 (Figure 4b). ${ }^{[51]}$

The relaxation measurements were performed below $150{ }^{\circ} \mathrm{C}$ for most networks (except CAN-1 and CAN-2) to prevent degradation of the TBD catalyst. ${ }^{[52]}$ In the case of CAN-1, an upper temperature of $170^{\circ} \mathrm{C}$ was used to increase the number of data points in the Arrhenius plot. For CAN-2, which was actually prepared as a reference material with the expectation of being an irreversible network, a slight stress relaxation was observed at $150{ }^{\circ} \mathrm{C}$ (Figure S6), which encouraged us to explore the limits of this material. Therefore, relaxation measurements were performed until an upper temperature as high as $240^{\circ} \mathrm{C}$ to probe the possible bond exchange via VTE-2-type moieties (vide supra). Indeed, full stress relaxation could be observed if enough energy was added to the system (Figure S6).
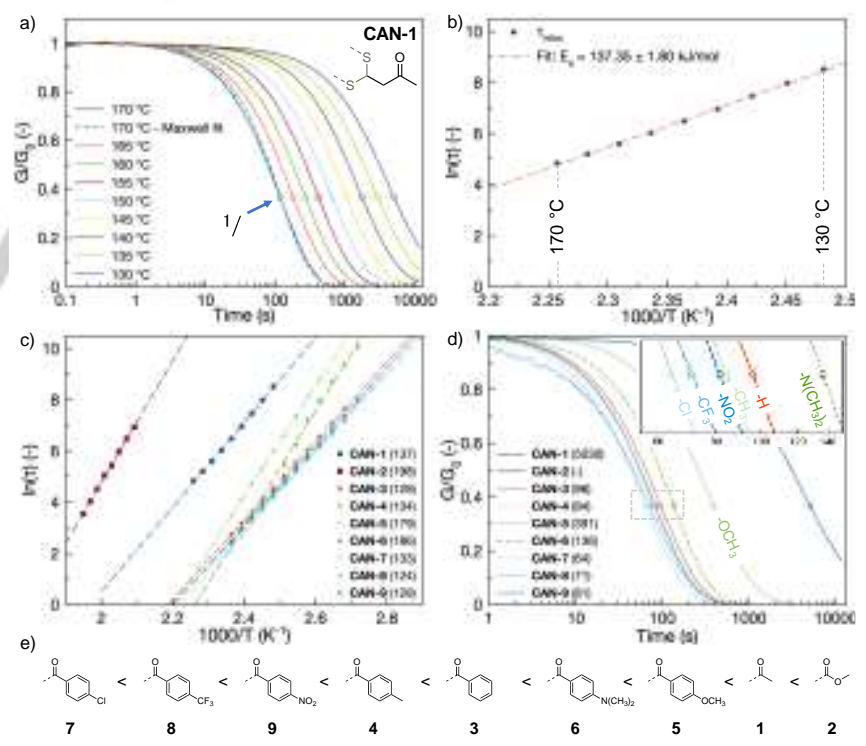

Figure 4. (a) Stress relaxation experiment of CAN-1 between 130 and $170{ }^{\circ} \mathrm{C}$ Relaxation times $(\tau)$ were determined at $\mathrm{G} / \mathrm{G}_{0}=1 / \mathrm{e}$. (b) Arrhenius plot of the obtained relaxation times with linear fit according to Arrhenius equation and the corresponding viscous flow activation energy $\left(E_{a}\right)$. (c) Overview of Arrhenius plots with linear fits for all networks. Values in brackets represent the activation energy in $\mathrm{kJ} / \mathrm{mol}$. (d) Overview of stress relaxation curves at $130{ }^{\circ} \mathrm{C}$ for all networks. Values in brackets represent the relaxation time (at 1/e) in seconds at $130{ }^{\circ} \mathrm{C}$. For CAN-6 a Maxwell fit (dashed line) was created for clear comparison. (e) Ranking of CANs with regard to relaxation time at $130{ }^{\circ} \mathrm{C}$. 
An overview of all Arrhenius plots is presented in Figure 4c. With the exception of $\mathbf{C A N}-6$, all networks exhibited a linear correlation of $\ln (\tau)$ with $1000 / T$ in the observed temperature window, indicating Arrhenius flow characteristics. The unusual stress relaxation curves of CAN-6 (Figure S10) were attributed to the absence of a rubbery plateau within this temperature range, which was confirmed by temperature sweep measurements (Figure S14). The Arrhenius plot of CAN-2 is situated on the far left of Figure $4 \mathrm{c}$, as very high temperatures were required to allow relaxation within a reasonable time frame. CAN-1 and CAN-3-9 showed relaxation in the same temperature range, however, the Arrhenius plot of CAN-1 was shifted to significantly longer relaxation times at a certain temperature compared to the phenylpropynone-derived materials. Similar activation energies in the range of $124-137 \mathrm{~kJ} / \mathrm{mol}$ were observed for CAN-1,3,4,7-9, whereas higher activation energies in the range of $179-198 \mathrm{~kJ} / \mathrm{mol}$ were observed for CAN-2,5,6. This difference is expected to originate from the presence of electronically deactivating groups (ester functionality in $\mathbf{C A N}-2$ or electron donating parasubstituents in CAN-5,6). Figure 4d displays an overview of stress relaxation curves at $130{ }^{\circ} \mathrm{C}$ of the networks to visualize the difference in relaxation times. Besides CAN-2 which did not show relaxation at $130{ }^{\circ} \mathrm{C}, \mathbf{C A N}-1$ showed the slowest relaxation at $130{ }^{\circ} \mathrm{C}(5030 \mathrm{~s})$, whereas the fastest relaxation was observed for CAN-7 (64 s).

The trends in exchange rate for different alkynone crosslinkers, as determined from kinetic model studies and DFT calculations, were not exactly reproduced on material level (Figure 4e). Moreover, the difference in stress relaxation times between phenylpropynone-derived materials (CAN-3-9) was not as pronounced as could be anticipated based on the remarkable differences in chemical relaxation times (Figure 1d). Nevertheless compared to the unsubstituted reference (CAN-3), electron withdrawing para-substituents still showed faster relaxation (CAN-7,8,9), whereas electron donating para-substituents exhibited slower relaxation (CAN-5,6), conserving the overall trends. Only CAN-4 did not follow this trend and showed a faster relaxation than the unsubstituted reference (CAN-3), but this effect can be attributed to an increased mobility in the matrix as also shown during DSC analysis $\left(T_{g}\right.$, CAN-3 $>T_{g}$, CAN-4 $)$. Similarly, the difference in $T_{g}$ could also explain the discrepancy in the ranking of CAN-7,8,9, where the high $T_{g}$ of CAN-9 indicates a restricted mobility, resulting in a slower relaxation time compared to $\mathbf{C A N - 7 , 8}$. These observations highlighted again the fact that exchange on a material level is not only controlled by the kinetics of dynamic bond exchange, but will also be substantially influenced by matrix effects, that are not captured by small molecule models. ${ }^{[3]}$

It is important to note that in the absence of an associative bond exchange mechanism, the prepared CANs cannot be classified as vitrimers, despite the observed linear relationship of relaxation times and temperature in the Arrhenius plots (Figure 4c). To illustrate the dissociative bond exchange mechanism operating in dynamic thiol-yne networks, frequency sweep measurements were performed at different temperatures. Whereas associative CANs or vitrimers are characterized by a constant plateau modulus $G^{\prime}$ at different temperatures as a result of their overall constant and temperature-independent crosslink density, dissociative CANs should show a decreasing $G^{\prime}$ with increasing temperature as a result of a net de-crosslinking. ${ }^{[5,53]}$ Figure 5 shows the frequency sweep measurements for CAN-1 and CAN-3 as representative networks. As expected, a temperature dependent decreasing plateau modulus was observed, nicely confirming the dissociative nature of the dynamic bond exchange. It is clear that in spite of this decrosslinking, none of the CANs, with exception of CAN-6 (Figure S10), go through a gel-to-sol transition within the probed temperature range, and there is thus no sudden drop in viscosity in the stress relaxation experiments.
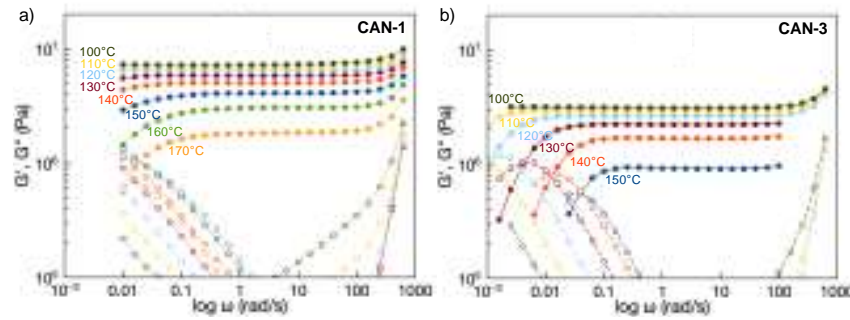

Figure 5. Frequency sweep measurements of (a) CAN-1 and (b) CAN-3. Full circles: G', empty circles: G".

Our small molecule models showed fast bond exchanges already happening at room temperature for most alkynones. At the other hand, the macroscopic exchange kinetics in the thiol-yne networks was much slower, with a much more pronounced temperature dependence (i.e., activation energy). To study the network integrity of the prepared CANs at lower to ambient temperatures, creep experiments are most suitable. These were performed on CAN-1 and CAN-3 as representative networks (Figure S15a,b). Both networks showed full shape recovery after deformations at 60 and $80^{\circ} \mathrm{C}$. However, a permanent deformation of both materials became prominent at temperatures above $100^{\circ} \mathrm{C}$, indicating activation of the dynamic chemistry. In line with previous observations in the stress relaxation experiments, the creep resistance of CAN-1 was better than the one of CAN-3 as a result of slower exchange. Also here, the relation between the creep rate $(\dot{\varepsilon})$ and temperature $(T)$ was fitted to the Arrhenius equation to retrieve the corresponding activation energy $\left(E_{a}\right)$ from the slope of the linear fit (Figure S16). ${ }^{[16]}$ The determined $E_{a}$ was similar for both CANs, but ca. $30 \mathrm{~kJ} / \mathrm{mol}$ lower than the $E_{a}$ obtained during stress relaxation experiments.

\section{Reprocessability of CANs}

Following the study on the viscoelastic properties of the dynamic networks, the mechanical properties of these materials upon reprocessing were analyzed. For this purpose, CAN-1 and CAN-3 were subjected to 5 cutting/reprocessing cycles using thermal compression molding at $140{ }^{\circ} \mathrm{C}$ for 30 minutes while monitoring their properties. Figure 6a,c shows the stress-strain curves of the original materials and after each reprocessing step. Since CAN-1 has a $T_{g}$ below room temperature, the material showed low stress and high strain, in contrast to $\mathbf{C A N}-3$, with a $T_{g}$ above room temperature. Upon reprocessing, the elongation, stress at break, and Young modulus remained very similar for CAN-1, proving 
adequate restoration of the original properties (Figure $7 \mathrm{~b}$ ). For CAN-3, a slight increase of the elongation and stress at break was observed (Figure 7d). The reprocessed materials also showed similar properties when monitored using swelling experiments (Table S1), DSC analysis (Figure S17), Fourier transform infrared spectroscopy (Figure S18) and Raman spectroscopy (Figure S19). Although the restoration of the original properties was successful in these recycling experiments, it was observed that $\mathbf{C A N}-1$ required slightly longer remolding times after the $3^{\text {rd }}$ reprocessing cycle in order to regenerate a sample with the same properties, which implied some type of ageing. In an attempt to accelerate and exaggerate thermal ageing effects upon reprocessing, consecutive stress relaxation measurements were performed at $170{ }^{\circ} \mathrm{C}$ on CAN-1 and CAN-3 (Figure S20-S21). Even though full relaxation was attained for both networks, an increased modulus upon each reprocessing cycle was observed, which was accompanied with an increased relaxation time. This effect can be readily rationalized by two factors, the first one being catalyst ageing (thermal degradation) leading to slower/less bond exchanges. Another explanation is that the networks become more perfect after reprocessing, as the virgin materials were found to still contain significant amounts of uncured VTEfunctions ( $10 \%$, vide supra).
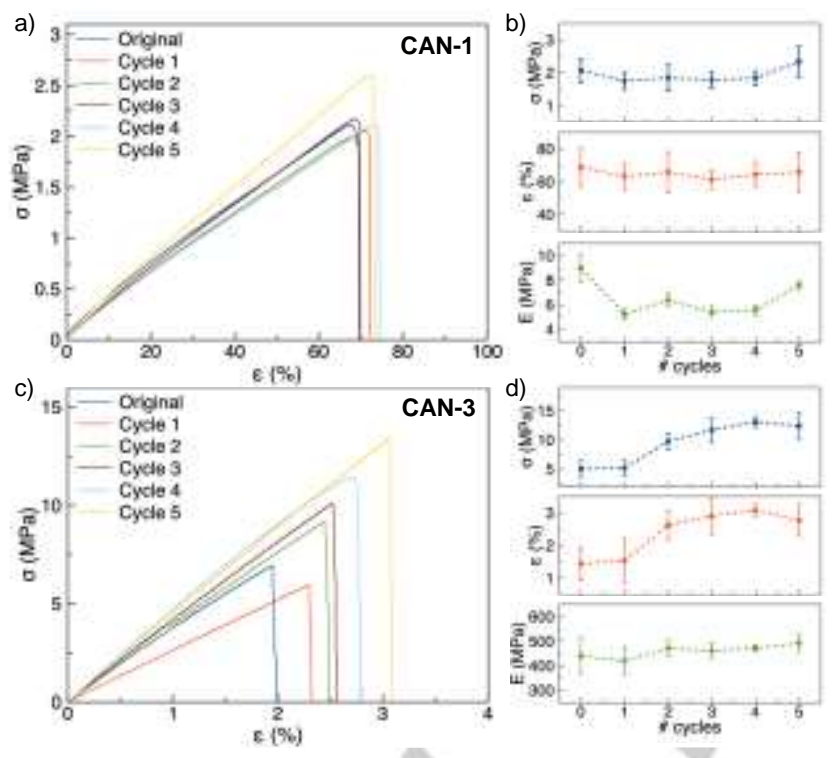

Figure 6. Overview of tensile strength experiments. (a) Representative stress strain curves of reprocessed CAN-1. (b) Young modulus (E), strain $(\varepsilon)$ and stress values $(\sigma)$ after each reprocessing cycle of CAN-1. (c) Representative stress-strain curves of reprocessed CAN-3. (d) Young modulus, strain and stress values after each reprocessing cycle of CAN-3.

\section{Conclusion}

This work demonstrates the use of simple activated alkyne species as versatile dynamic cross-linkers for thiol-yne based polymer networks, resulting in a promising new chemical platform for the design of covalent adaptable networks. A wide series of alkynone precursors was explored for their click-like cross-linking reactivity that can join two thiols into a dynamic thioacetal linkage. Kinetic model studies revealed that exchange rates were significantly influenced by the steric and electronic nature of the alkyne cross-linker, with exchange rate constants spanning several orders of magnitude. Diverse substituents on a phenylpropynone scaffold showed a clear trend in rate effects, showing faster exchange rates for electron withdrawing substituents, and slower rates for electron donating groups. This behavior is in line with mechanistic expectations and was also further rationalized by density functional theory calculations.

The dynamic thiol-yne platform was subsequently implemented in materials to produce CANs. An extensive rheological analysis was performed to probe the interdependence of chemical structure and the dynamic network properties. A remarkable difference was observed in macroscopic network relaxation times, compared to the 'chemical' exchange rates observed in small molecule models. Whereas the chemical exchange is already quite dynamic at or just above room temperature in small molecule models, the corresponding thiol-yne networks proved to be much less reactive, also confirmed by creep experiments. This demonstrates that overreliance on chemical model reactions for the design of CANs may be a misleading approach. Indeed, the thermodynamic landscape of bond exchange pathways can be quite dramatically altered by the macromolecular matrix and context.

The prepared dynamic networks showed excellent reprocessability and overall network integrity as proven by mechanical and thermal characterization, showing that this new chemistry platform could be further explored in many application areas for which CANs are being investigated. Not only can this reversible chemistry be used for reversible cross-linking in the context of CANs, but also in other dynamic applications and concepts, such as reversible functionalization, reversible polymerization, dynamic combinatorial libraries, etc. Especially the double functionality of the activated yne can lead to interesting dynamic applications and new concepts. In conclusion, this platform opens up many new avenues for further research within the field of CANs.

\section{Acknowledgements}

N.V.H. and M.G. acknowledge the Research Foundation Flanders (Fonds Wetenschappelijk Onderzoek - Vlaanderen) for Ph.D. and Postdoctoral fellowships. K.U. acknowledges UGent for funding. F.D.P acknowledges UGent funding (BOF-GOA). B. De Meyer, J. Goeman and T. Courtin are thanked for technical support. The computational resources (Stevin Supercomputer Infrastructure) and services used in this work were provided by the VSC (Flemish Supercomputer Center), funded by Ghent University, FWO and the Flemish Government - department EWI.

Keywords: Thiol-yne $\cdot$ Retro reactions $•$ Dynamic chemistry • Covalent adaptable network $\cdot$ Thioacetal 
[1] C. J. Kloxin, C. N. Bowman, Chem. Soc. Rev. 2013, 42, 7161-7173.

[2] G. M. Scheutz, J. J. Lessard, M. B. Sims, B. S. Sumerlin, J. Am. Chem. Soc. 2019, jacs.9b07922.

[3] J. Winne, L. Leibler, F. E. Du Prez, Polym. Chem. 2019, Accepted.

[4] M. K. McBride, B. T. Worrell, T. Brown, L. M. Cox, N. Sowan, C. Wang, M. Podgorski, A. M. Martinez, C. N. Bowman, Annu. Rev. Chem. Biomol. Eng. 2019, 10, 175-198.

[5] C. J. Kloxin, C. N. Bowman, Chem. Soc. Rev. 2013, 42, 7161-73.

[6] P. Chakma, D. Konkolewicz, Angew. Chemie Int. Ed. 2019, 58, 9682 9695.

[7] W. Denissen, J. M. Winne, F. E. Du Prez, Chem. Sci. 2016, 7, 30-38.

[8] X. Chen, M. A. Dam, K. Ono, A. Mal, H. Shen, S. R. Nutt, K. Sheran, F. Wudl, Science 2002, 295, 1698-1702.

[9] Y. Higaki, H. Otsuka, A. Takahara, Macromolecules 2006, 39, 21212125.

[10] S. Billiet, K. De Bruycker, F. Driessen, H. Goossens, V. Van Speybroeck, J. M. Winne, F. E. Du Prez, Nat. Chem. 2014, 6, 815-821.

[11] H. A. Houck, K. De Bruycker, C. Barner-Kowollik, J. M. Winne, F. E. Du Prez, Macromolecules 2018, 51, 3156-3164.

[12] N. Van Herck, F. E. Du Prez, Macromolecules 2018, 51, 3405-3414.

[13] M. Delahaye, J. M. Winne, F. E. Du Prez, J. Am. Chem. Soc. 2019, 141, 15277-15287.

[14] D. Montarnal, M. Capelot, F. Tournilhac, L. Leibler, Science 2011, 334, 965-968.

[15] W. Denissen, G. Rivero, R. Nicolaÿ, L. Leibler, J. M. Winne, F. E. Du Prez, Adv. Funct. Mater. 2015, 25, 2451-2457.

[16] M. Guerre, C. Taplan, R. Nicolaÿ, J. M. Winne, F. E. Du Prez, J. Am. Chem. Soc. 2018, 140, 13272-13284.

[17] Y. Spiesschaert, M. Guerre, L. Imbernon, J. M. Winne, F. Du Prez, Polymer 2019, 172, 239-246.

[18] C. Taplan, M. Guerre, J. M. Winne, F. E. Du Prez, Mater. Horizons 2019, 10.1039/C9MH01062A.

[19] B. Hendriks, J. Waelkens, J. M. Winne, F. E. Du Prez, ACS Macro Lett. 2017, 6, 930-934.

[20] M. M. Obadia, A. Jourdain, P. Cassagnau, D. Montarnal, E. Drockenmuller, Adv. Funct. Mater. 2017, 27, 1703258.

[21] T. F. Scott, A. D. Schneider, W. D. Cook, C. N. Bowman, Science 2005, 308, 1615-1617.

[22] W. A. Ogden, Z. Guan, J. Am. Chem. Soc. 2018, 140, 6217-6220.

[23] B. Zhang, Z. A. Digby, J. A. Flum, P. Chakma, J. M. Saul, J. L. Sparks, D. Konkolewicz, Macromolecules 2016, 49, 6871-6878.

[24] J. S. A. Ishibashi, J. A. Kalow, ACS Macro Lett. 2018, 7, 482-486.

[25] G. Joshi, E. V Anslyn, Org. Lett. 2012, 14, 4714-4717.

[26] B. M. Matysiak, P. Nowak, I. Cvrtila, C. G. Pappas, B. Liu, D. Komaromy, S. Otto, J. Am. Chem. Soc. 2017, 139, 6744-6751.

[27] P. Chakma, L. H. Rodrigues Possarle, Z. A. Digby, B. Zhang, J. L. Sparks, D. Konkolewicz, Polym. Chem. 2017, 8, 6534-6543.

[28] S. P. Daymon, K. M. Miller, Polymer 2018, 145, 286-293.

[29] N. Kuhl, R. Geitner, R. K. Bose, S. Bode, B. Dietzek, M. Schmitt, J. Popp, S. J. Garcia, S. van der Zwaag, U. S. Schubert, et al., Macromol. Chem. Phys. 2016, 217, 2541-2550.

[30] B. T. Worrell, S. Mavila, C. Wang, T. M. Kontour, C.-H. Lim, M. K. McBride, C. B. Musgrave, R. Shoemaker, C. N. Bowman, Polym. Chem. 2018, 9, 4523-4534.

[31] S.-H. Shim, M. Ham, J. Huh, Y.-K. Kwon, Y.-J. Kwark, Polym. Chem. 2013, 4, 5449.

[32] L. J. Macdougall, V. X. Truong, A. P. Dove, ACS Macro Lett. 2017, 6, 93-97.

[33] A. B. Lowe, Polymer 2014, 55, 5517-5549.

[34] D. P. Nair, M. Podgórski, S. Chatani, T. Gong, W. Xi, C. R. Fenoli, C. N. Bowman, Chem. Mater. 2014, 26, 724-744.

[35] H. Kuroda, I. Tomita, T. Endo, Polymer 1997, 38, 6049-6054.

[36] O. Daglar, U. S. Gunay, G. Hizal, U. Tunca, H. Durmaz, Macromolecules 2019, 52, 3558-3572.

[37] C. Xu, J. K. Bartley, D. I. Enache, D. W. Knight, M. Lunn, M. Lok, G. J. Hutchings, Tetrahedron Lett. 2008, 49, 2454-2456.
[38] M. Zheng, F. Wu, K. Chen, S. Zhu, Org. Lett. 2016, 18, 3554-3557.

[39] G. Ieronimo, G. Palmisano, A. Maspero, A. Marzorati, L. Scapinello, N. Masciocchi, G. Cravotto, A. Barge, M. Simonetti, K. L. Ameta, et al., Org Biomol. Chem. 2018, 16, 6853-6859.

[40] J. M. Wilbur, B. A. Bonner, J. Polym. Sci. Part A Polym. Chem. 1990, 28, 3747-3759

[41] V. X. Truong, A. P. Dove, Angew. Chemie Int. Ed. 2013, 52, 4132-4136.

[42] D.-J. Zhang, M.-S. Xie, G.-R. Qu, Y.-W. Gao, H.-M. Guo, Org. Lett. 2016 18, 820-823.

[43] Y. S. Lee, K. H. Chung, Y. H. Kim, Heteroat. Chem. 1999, 10, 461-464.

[44] Y. Zhong, Y. Xu, E. V. Anslyn, European J. Org. Chem. 2013, 50175021.

[45] B. Zhang, P. Chakma, M. P. Shulman, J. Ke, Z. A. Digby, D. Konkolewicz, Org. Biomol. Chem. 2018, 16, 2725-2734.

[46] E. H. Krenske, R. C. Petter, K. N. Houk, J. Org. Chem. 2016, 81, 1172611733.

[47] M. Capelot, M. M. Unterlass, F. Tournilhac, L. Leibler, ACS Macro Lett. 2012, 1, 789-792.

[48] J. Van Damme, O. van den Berg, J. Brancart, L. Vlaminck, C. Huyck, G. Van Assche, B. Van Mele, F. Du Prez, Macromolecules 2017, 50, 1930 1938

[49] P. J. Flory, J. Am. Chem. Soc. 1941, 63, 3083-3090.

[50] W. H. Stockmayer, J. Chem. Phys. 1943, 11, 45-55.

[51] L. H. Sperling, Introduction to Physical Polymer Science, John Wiley \& Sons, Inc., Hoboken, NJ, USA, 2005.

[52] C. Jehanno, I. Flores, A. P. Dove, A. J. Müller, F. Ruipérez, H. Sardon, Green Chem. 2018, 20, 1205-1212.

[53] P. J. Flory, Chem. Rev. 1944, 35, 51-75. 


\section{Entry for the Table of Contents}

\section{RESEARCH ARTICLE}

Controlling reactivity: Reversible thiol-yne cross-linking is enabled via the double Michael addition of thiols on activated alkynes. The rate of dynamic thiol exchange is manipulated by means of electronic substituents on the activated alkyne, offering a new versatile dynamic platform for the development of covalent adaptable networks.

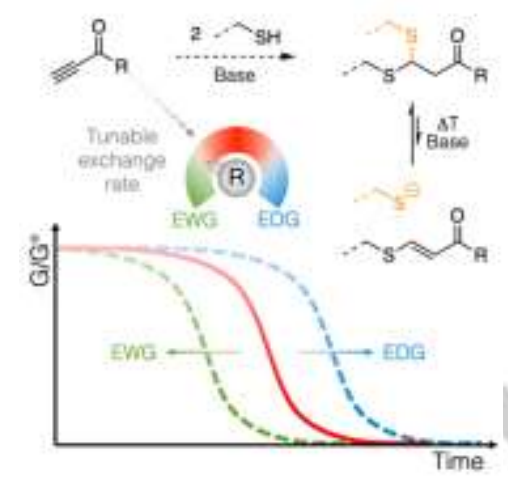

Niels Van Herck, Diederick Maes, Kamil Unal, Marc Guerre, Johan M. Winne*, Filip E. Du Prez*

Page No. - Page No.

Covalent adaptable networks with tunable exchange rates based on reversible thiol-yne cross-linking 